\section{Ibrutinib-associated skin toxicity: a case of maculopapular rash in a 79-year old Caucasian male patient with relapsed Waldenstrom's macroglobu- linemia and review of the literature}

\author{
Anders Bisgaard Jensen, ${ }^{1}$ \\ Birgitte Stausbøl-Grøn, ${ }^{2}$ \\ Rikke Riber-Hansen, ${ }^{3}$ \\ Francesco d'Amore ${ }^{1}$ \\ ${ }^{1}$ Department of Hematology; \\ ${ }^{2}$ Department of Dermatology; \\ ${ }^{3}$ Department of Pathology, Aarhus \\ University Hospital, Aarhus, Denmark
}

\begin{abstract}
Waldenstrom's macroglobulinamia (WM) is a rare malignant lymphoproliferative disorder, characterized by monoclonal IgM paraproteinemia and neoplastic proliferation of malignant lymphoplasmacytoid cells in the bone marrow. Traditionally, WM has been treated with modalities similar to those used in the management of other indolent lymphomas. Just recently, based on impressive clinical trial results in heavily pretreated WM patients, a new Bruton Tyrosine Kinase-inhibitor, Ibrutinib, has been approved for the treatment of this disorder. As the use of Ibrutinib in WM outside clinical trials is still limited, only few clinical reports illustrating treatment side effects are currently available. Here we review the current literature specific on Ibrutinib-associated rash in hematologic patients, and report on an elderly patient with WM, who developed a red maculopapular non-pruritic rash 12 weeks after starting Ibrutinib therapy. Without modifications of the ongoing Ibrutinib schedule, the rash regressed within two weeks of treatment with topical steroidcontaining dermatological compounds.
\end{abstract}

\section{Introduction}

As in other indolent lymphoproliferative malignancies, not all WM patients require treatment at the time of diagnosis. Most often these patients' disease status will be followed and cytoreductive intervention suggested if clinically indicated. In patients where therapeutic intervention is deemed necessary Rituximab-based combination therapy regimens currently remains the therapy of choice. Monotherapy with Rituximab is only moderately effective with overall response rates (ORR) of $40-50 \%$ in previously untreated patients. ${ }^{1}$ Combinations of Rituximab with conventional cytotoxic agents, such as Cyclophosphamide and Dexamethasone (RCD) or Bendamustine (BR), increase ORR up to $80-95 \%$ and median progression-free survival (PFS) values to $35-70$ months. ${ }^{2,3}$

A growing number of novel drugs are currently undergoing clinical trials in WM. One of these is the Bruton's tyrosine kinase (BTK) inhibitor Ibrutinib, recently approved for the treatment of WM. In WM, a MYD88 ${ }^{\mathrm{L} 265 \mathrm{P}}$ mutation has been proven in 93-97\% of patients. ${ }^{4}$ This gain-of-function mutation activates the BTK pathway, which in turn increases $\mathrm{NF}-\kappa \mathrm{B}$ signaling. ${ }^{5}$ Ibrutinib interferes with this B-cell proliferation signal by inhibiting the BCR, BTK pathway, ultimately leading to increased apoptosis as demonstrated in chronic lymphatic leukemia (CLL) cells. ${ }^{6}$

Several clinical trials with Ibrutinib have proven the drug to be effective in Bcell malignancies such as mantle-cell lymphoma (MCL) and CLL. A recent clinical trial has shown Ibrutinib treatment to be highly effective in WM as well, inducing durable responses in a group of 63 pretreated WM patients, with an ORR of $90.5 \%$ along with 2 year PFS and OS of $69.1 \%$ and $95.2 \%$, respectively. ${ }^{7}$ Overall the current results of Ibrutinib treatment are looking very promising when compared to previously used regimens of Rituximab as monotherapy or regimens of Rituximab combined with conventional cytotoxic agents. ${ }^{8}$ The recent clinical Ibrutinib trial found grade $\geq 3$ adverse events to be neutropenia $(14 \%)$ and thrombocytopenia $(13 \%)$. Less frequent $(\leq 5 \%)$ were cardiac (e.g., arrhythmia), gastrointestinal (diarrhea) and hemorrhagic side effects. Skin events including pruritus, rash and skin exfoliation were observed in $2 \%$ of patients. Case reports specifically focusing on skin reactions to Ibrutinib treatment are described in CLL and MCL patients. ${ }^{9,10}$ More recently, Iberri et al. reported on Ibrutinib-related skin rashes in CLL and MCL patients. The authors describe two types of rash presentations: a non-palpable, largely asymptomatic rash, and a palpable, pruritic type. ${ }^{11}$ No detailed clinical descriptions of Ibrutinib associated skin rash have yet been reported in patients with WM.
Correspondence: Anders Bisgaard Jensen, Department of Hematology, Aarhus University Hospital, Aaparken 1, 0-15, 8000 Aarhus C, Denmark. Tel: +45.25566176 .

E-mail: ajensen@biomed.au.dk

Key words: Waldenstrom's macroglobulinamia; ibrutinib; rash; adverse effects; minireview.

Contributions: $\mathrm{ABJ}$ and $\mathrm{FdA}$, collected data along with patient case information and interpreted these, reviewed the literature on the subject. BS-G produced clinical images and assisted with dermatological expertise, interpretation and description of this. RR-H produced, interpreted and described the histological samples. Drafting, critical revision and final approval of the manuscript was undertaken by all authors.

Conflict of interest: the authors declare no potential conflict of interest.

Received for publication: 17 November 2016 Accepted for publication: 31 March 2017

This work is licensed under a Creative Commons Attribution-NonCommercial 4.0 International License (CC BY-NC 4.0).

(C) Copyright A.B. Jensen et al., 2017

Licensee PAGEPress, Italy

Dermatology Reports 2017; 9:6976

doi:10.4081/dr.2017.6976

\section{Case Report}

A 79-year-old man with WM diagnosed in 2002 presented to our out-patient clinic for evaluation of increasing pancytopenia (total white blood cell count $2.9 \times 10^{9} / \mathrm{L}$, absolute neutrophil count $1.61 \times 10^{9} / \mathrm{L}$; Hemoglobin $10.63 \mathrm{~g} / \mathrm{dL}$, platelet count $\left.146 \times 10^{9} / \mathrm{L}\right)$ and $\operatorname{IgM}$ paraproteinemia $(3500$ $\mathrm{mg} / \mathrm{dL}$ ) associated with fatigue, drenching night sweats, recurrent bacterial and viral infections and hypogammaglobinemia. A bone marrow biopsy revealed marrow compartments heavily infiltrated (approximately $90 \%$ of the evaluated marrow cellularity) by a homogeneous population of lymphoplasmacytoid cells morphologically and immunohistochemically (CD2+, CD79a+, intracytoplasmic $\kappa$-light chain restriction) compatible with WM. The patient had previously been treated with a number of regimens including a combination of rituximab + cyclophosphamide + vincristine + prednisone (R-CVP) in 2004, as well as fludarabine (R-FC) in 2009, rituximab + bendamustine (BR) in 2012, bortezumib + rituximab + dexamethasone (BDR) in 2015, 
with the most recent therapy given 5 months prior to his out-patient clinic visit. $\mathrm{He}$ was started on Ibrutinib $420 \mathrm{mg}$ PO daily. The patient continued with two medications he had been receiving over several months prior to Ibrutinib, i.e. human $\operatorname{IgG}$ immunoglobulin (SC) and Allopurinol $300 \mathrm{mg}$ (PO) once daily.

Twelve weeks after starting Ibrutinib treatment, the patient developed a painless, slightly pruritic rash starting from the groins and wrists and subsequently spreading to trunk, extremities and with single elements also in the facial area (Figure 1). The rash was characterized by a diffuse distribution of bright red edematous maculopapular elements, ranging from 0.5 to $1 \mathrm{~cm}$ in diameter, with sparing of his palms and soles (grade 3 according to NCI-CTC). Never before had the patient experienced a similar rash, nor had any of his family members or close relations similar symptoms concomitant to the time of onset in the patient. With regard to $\mathrm{WM}$, the patient had a good clinical response to Ibrutinib, with IgM values gradually dropping to normal levels within the first two- three months from treatment start. Absolute eosinophil count at the time of rash onset was within normal range (0.2- $\left.0.5 \times 10^{9} / \mathrm{L}\right)$. Blood work showed no significant elevation of liver enzymes or signs of impaired kidney function, which would suggest a more systemic reaction to the drug. After an initial clinical assessment, the patient was referred to the department of dermatology, where a punch biopsy from a relevant rash area was obtained for histological evaluation. This biopsy showed lymphocytic infiltration with numerous eosinophil granulocytes, mainly in the superficial dermal layer, but also in the perivascular- and interstitial tissue (Figure 2A). A PAS staining of the sample was negative for fungi (Figure 2B). These findings, integrated with the clinical picture and the therapeutic history, led to the diagnosis of drug-induced rash. Topical steroid treatment was initiated under dermatological supervision. While effective in eliminating the pruritus symptoms, topical treatment did not lead to a rapid resolution of the maculopapular elements. Tapering of Ibrutinib dose was considered, but not implemented in the absence of systemic and cutaneous clinical progression. At subsequent clinical evaluations, under persistent topical treatment, the rash gradually subsided and eventually resolved approximately 3 months after its onset. At last follow-up, 6 months after rash resolution, the patient is still on Ibrutinib and has not experienced reoccurrence of the cutaneous manifestations.

Written consent from the patient has been obtained.

\section{Discussion}

Ibrutinib (Imbruvica, Janssen Biotech), an oral BTK inhibitor is approved for use in refractory CLL and MCL. In 2015, Ibrutinib was also approved for use in pre-treated adult WM patients, or previously untreated WM patients not eligible for chemotherapy. Ibrutinib has been shown to have good clinical and paraclinical effect in these patients, and is generally very well tolerated with a low frequency of severe adverse events. ${ }^{12-14}$

In recently published results, from early phase clinical trials testing Ibrutinib as monotherapy in MCL, CLL and small lymphocytic lymphoma (SLL), rash occurred at a frequency of $13-27 \% .{ }^{9,10,13-15}$ A recent study reporting the Stanford University experience with Ibrutinib-associated rash development in MCL and CLL patients described two different types of clinical pic-

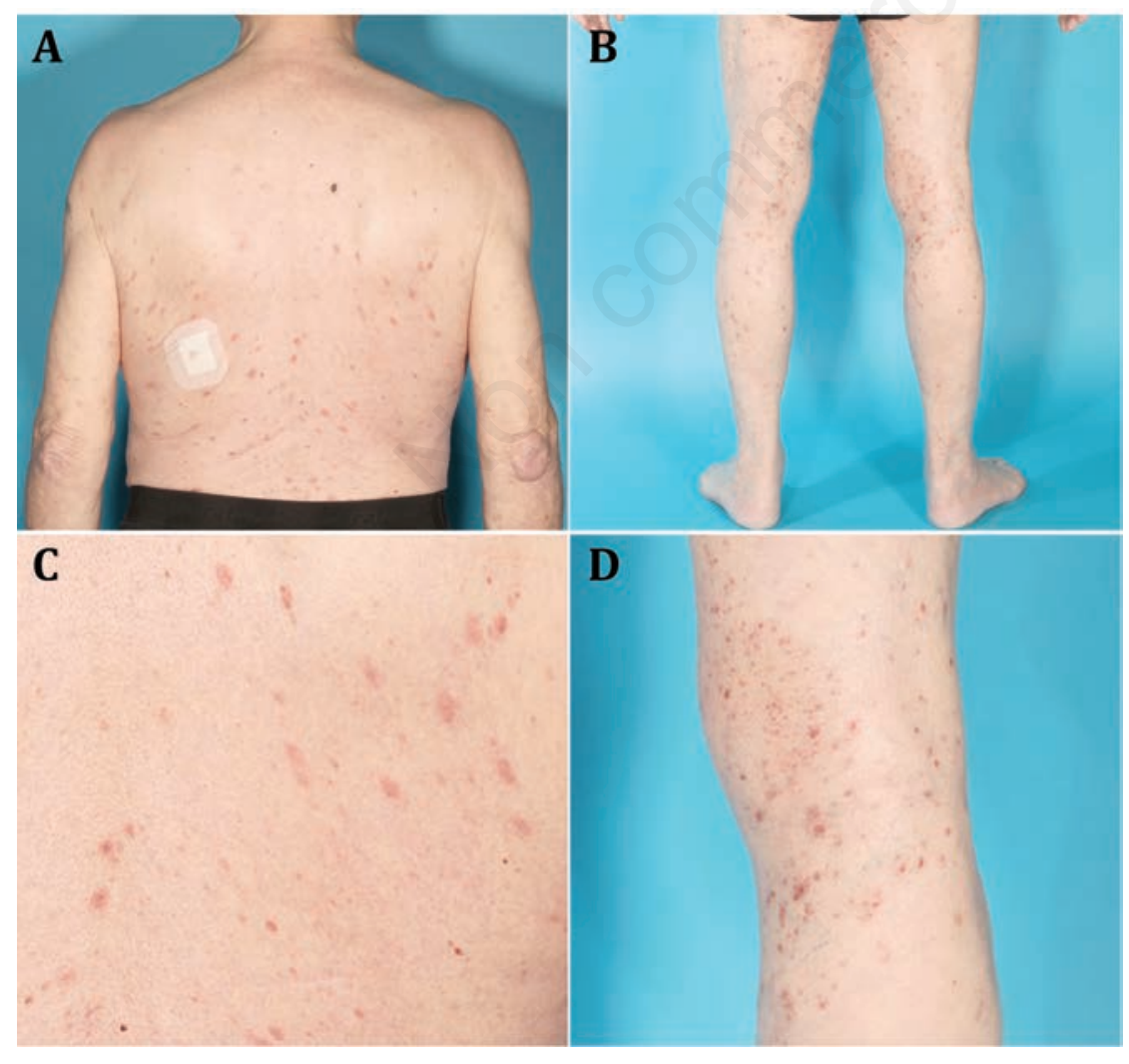

Figure 1. Pruritic rash in Waldenstrom's patient developed 12 weeks after beginning Ibrutinib therapy. A) Upper back; B) back thighs and calves; C and D) close-up of rash papules seen in panels $\mathrm{A}$ and $\mathrm{B}$.

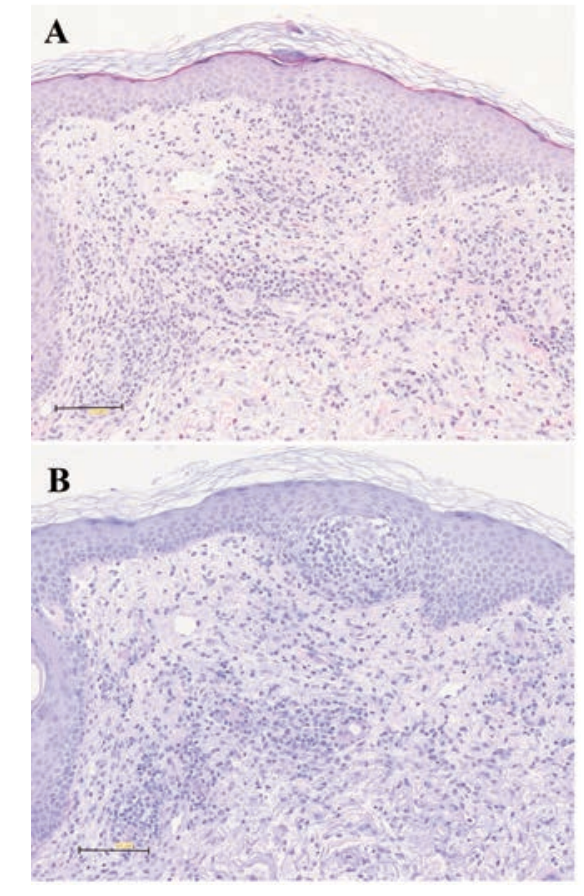

Figure 2. A slightly spongiotic reaction with predominantly perivascular infiltrates of lymphocytes and eosinophils with a negative reaction for fungi were seen in the histopathological examination of the punch biopsy of the skin (A: Hematoxylin Eosin, x200; B: Periodic Acid-Schiff, x200). 
tures: i) a non-palpable, late-onset mild cutaneous eruption not requiring skindirected therapy or Ibrutinib tapering; and ii) a palpable purpuric rash, with earlier onset, generally more severe, requiring skin-directed intervention with topical therapy and oral antihistamines. ${ }^{11}$ In half of the patients with grade 3 rashes, Ibrutinib was either tapered or temporarily halted. All patients were able to resume Ibrutinib treatment at full dose or (in two patients) at a permanently dose reduced level. Another group reported of an IgG LPL patient who developed a rash consistent with Schnitzler's syndrome. However rash onset, look and symptoms in our patient shared only few similar characteristics with the typical presentation of Schnitzlers, and thus the rash was not suspected a part of this specific syndrome. Apart from being a BTKinhibitor, Ibrutinib has been shown to effectively inhibit the epidermal growth factor receptor (EGFR) in a dose dependent manner. ${ }^{16}$ Inhibition of EGFR is known to stimulate apoptosis, inflammation, enhance apoptosis and inhibit cell cycle progression. ${ }^{17}$ Cutaneous eruptions are a well-known adverse effect to EGFR inhibition by other tyrosine kinase inhibitors (TKI), ${ }^{18}$ and similarly Ibrutinib-induced rash may in part be a result of EGFR inhibition. The time of onset, and the general appearance of the rash could resemble a late-phase IgE-mediated reaction. The histological sample with lymphocytic and eosoniphile infiltration supports this theory, although the patient had not experienced any immediate reaction to the compound, and had not previously experienced atopic reactions to other drugs. Another possible explanation is that the rash represents a viral reactivation as a result of the patient's immunocompromised state. If this were the case, it would be the first time the patient experienced viral reactivation during treatment since diagnosed in 2002. No further investigation of the underlying mechanism was made, as the rash gradually resolved on topical steroid treatment. Data on skinbased toxicity in WM patients are largely lacking, and as of now only a small amount of detailed clinical characteristics of rash in this group of patients have been described. It is therefore only possible to compare this presentation to other patient groups. Ibrutinib-induced rashes have been described in MCL, CML and CLL patients, all receiving 400-600 $\mathrm{mg}$ of Ibrutinib (Figure 3). ${ }^{9,11,19,20}$ As can be seen from these reports, the time of rash onset is highly variable, with onset as late as 300-400 days after starting treatment, in some patients. The rash and pruritus symptoms in our patient are comparable to the symptoms described in the MCL/CLL group II. Compared to this group, rash onset is delayed in our patient ( 84 days compared to median 15 days), and no Ibrutinib tapering or pausing was deemed necessary.

\section{Conclusions}

Here we report of an elderly WM patient who developed a rash 84 days after initiating Ibrutinib therapy. This is, to our knowledge the first case description of an Ibrutinib-associated rash in a WM patient. The possible mechanism could be attributed to either EGFR action of the compound, $\operatorname{IgE}$ mediated allergic reaction or more simply, a viral reactivation caused by immunocomprimisation. In comparison to the other patient groups, rash onset was delayed and symptoms were milder when compared to the other cases presented with pruritic symptoms, and comparable CLL/MCL type II rash group of the Stanford study. Due to the novelty of the drug in the WM setting, detailed, and disease-specific clinical descriptions of toxicity profiles in general, and skin-based in particular, are valuable information for daily clinical practice.

By providing a detailed report on the clinical manifestation and subsequent management of Ibrutinib-associated skin-toxicity in a WM patient, our case report contributes in generating a shared clinical experience useful for recognition and management of Ibrutinib-associated rash. The relevance of this data is underscored by the increasing use of Ibrutinib in WM, after its recent approval.

\section{References}

1. Treon SP, Emmanouilides C, Kimby E, et al. Extended rituximab therapy in Waldenstrom's macroglobulinemia. Ann Oncol 2005;16:132-8.

2. Rummel MJ, Niederle N, Maschmeyer $\mathrm{G}$, et al. Bendamustine plus rituximab versus CHOP plus rituximab as firstline treatment for patients with indolent and mantle-cell lymphomas: an openlabel, multicentre, randomised, phase 3 non-inferiority trial. Lancet 2013;381: 1203-10.

3. Dimopoulos MA, Anagnostopoulos A, Kyrtsonis MC, et al. Primary treatment of Waldenstrom macroglobulinemia with dexamethasone, rituximab, and cyclophosphamide. J Clin Oncol 2007; 25:3344-9.

4. Treon SP, Xu L, Hunter Z. MYD88 Mutations and Response to Ibrutinib in Waldenstrom's Macroglobulinemia. N Engl J Med 2015;373:584-6.
Figure 3. An overview of rash onset from previously published studies and case reports regarding Ibrutinib-associated rashes in CML, CLL and MCL patients. References refer to reference list.

\section{Days of treatment till rash onset}

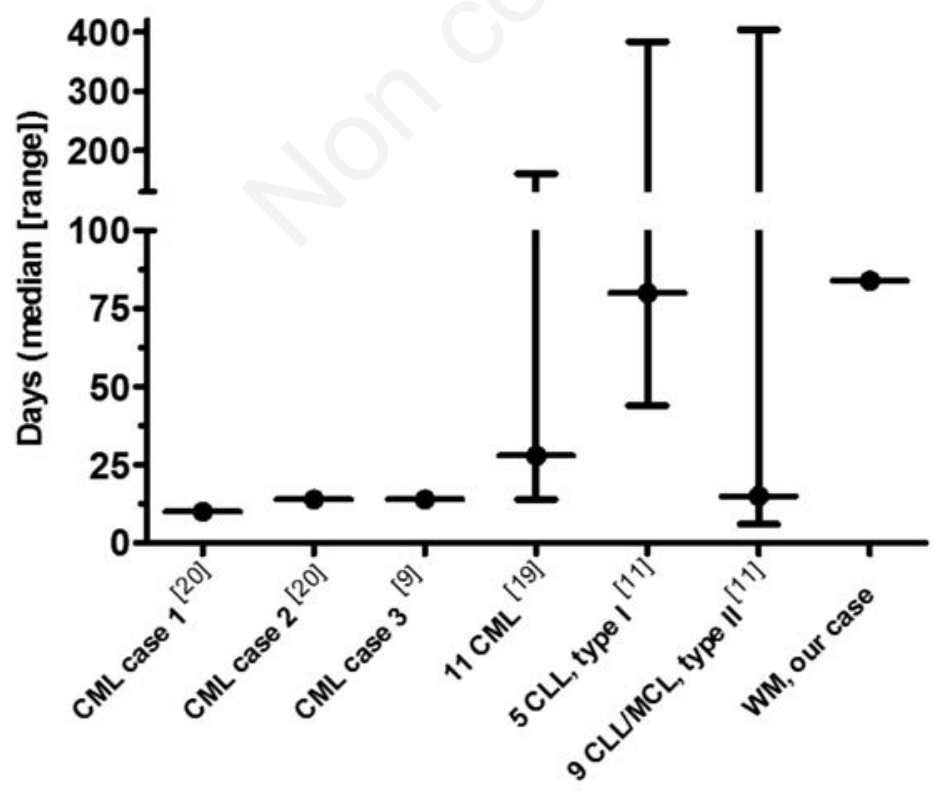


5. Treon SP, Xu L, Yang G, et al. MYD88 L265P somatic mutation in Waldenstrom's macroglobulinemia. N Engl J Med 2012;367:826-33.

6. Herman SE, Gordon AL, Hertlein E, et al. Bruton tyrosine kinase represents a promising therapeutic target for treatment of chronic lymphocytic leukemia and is effectively targeted by PCI32765. Blood 2011;117:6287-96.

7. Treon SP, Tripsas CK, Meid K, et al. Ibrutinib in previously treated Waldenstrom's macroglobulinemia. N Engl J Med 2015;372:1430-40.

8. Castillo JJ, Palomba ML, Advani R, Treon SP. Ibrutinib in Waldenstrom macroglobulinemia: latest evidence and clinical experience. Ther Adv Hematol 2016;7:179-86.

9. Mannis G, Wu D, Dea T, et al. Ibrutinib rash in a patient with $17 \mathrm{p}$ del chronic lymphocytic leukemia. Am J Hematol 2015;90:179.

10. Tobinai K, Ogura M, Ishizawa K, et al. Safety and tolerability of ibrutinib monotherapy in Japanese patients with relapsed/refractory B cell malignancies. Int J Hematol 2016;103:86-94.

11. Iberri DJ, Kwong BY, Stevens LA, et al. Ibrutinib-associated rash: a single-centre experience of clinicopathological features and management. $\mathrm{Br} \mathrm{J}$ Haematol 2016 [Epub ahead of print].

12. Advani RH, Buggy JJ, Sharman JP, et al. Bruton tyrosine kinase inhibitor ibrutinib (PCI-32765) has significant activity in patients with relapsed/refractory B-cell malignancies. J Clin Oncol 2013;31:88-94.

13. Byrd JC, Furman RR, Coutre SE, et al. Targeting BTK with ibrutinib in relapsed chronic lymphocytic leukemia. N Engl J Med 2013;369:32-42.

14. O'Brien S, Furman RR, Coutre SE, et al. Ibrutinib as initial therapy for elderly patients with chronic lymphocytic leukaemia or small lymphocytic lymphoma: an open-label, multicentre, phase 1b/2 trial. Lancet Oncol 2014; 15:48-58.

15. Wang ML, Rule S, Martin P, et al. Targeting BTK with ibrutinib in relapsed or refractory mantle-cell lymphoma. N Engl J Med 2013;369:50716.

16. Chen J, Kinoshita T, Sukbuntherng J, et al. Ibrutinib Inhibits ERBB Receptor Tyrosine Kinases and HER2-Amplified Breast Cancer Cell Growth. Endocr Relat Cancer 2016;15:689-708.

17. Woodworth CD, Michael E, Marker D, et al. Inhibition of the epidermal growth factor receptor increases expression of genes that stimulate inflammation, apoptosis, and cell attachment. Mol Cancer Ther 2005;4:650-8.

18. Perez-Soler R, Van Cutsem E. Clinical research of EGFR inhibitors and related dermatologic toxicities. Oncology (Williston Park) 2007;21:10-6.

19. Milojkovic D, Short K, Salisbury JR, et al. Dose-limiting dermatological toxicity secondary to imatinib mesylate (STI571) in chronic myeloid leukaemia. Leukemia 2003;17:1414-6.

20. Rule SA, O'Brien SG, Crossman LC. Managing cutaneous reactions to imatinib therapy. Blood 2002;100:3434-5. 